\title{
Choosing Wisely: five recommendations related to tests, treatments, and procedures at risk of inappropriateness in the cure of Parkinson's disease (LIMPE-DISMOV Academy)
}

\author{
Laura Avanzino ${ }^{1,2}$ (D) Pietro Cortelli ${ }^{3,4}$ on behalf of the Board of the LIMPE-DISMOV Academy
}

Received: 7 December 2018 / Accepted: 8 February 2019 / Published online: 14 February 2019

(C) The Author(s) 2019

Dear Editor,

We are reporting five recommendations related to tests, treatments, and procedures at risk of inappropriateness in the cure of Parkinson's disease. These recommendations were identified by the Academy for the Study of Parkinson's Disease and Movement Disorders (LIMPE-DISMOV Academy), in collaboration with Slow Medicine. Slow Medicine, an Italian movement of health professionals, patients, and citizens promoting a "Measured, Respectful and Equitable Medicine", launched the campaign "Doing more does not mean doing better- Choosing Wisely Italy" in Italy at the end of 2012, similar to Choosing Wisely in the USA. The campaign aims to help physicians, other health professionals, patients, and citizens engage in conversations about tests or procedures commonly used in their field whose necessity should be questioned and discussed. This call to action has resulted in specialty-specific lists of "Things Providers and Patients Should Question."

The five recommendations related to Parkinson's disease were selected during a meeting of the board of the LIMPEDISMOV Academy on the basis of a list drawn up by the individual members of the board. Each member of the board has indicated a practice, commonly carried out in Italy, for

Laura Avanzino

lavanzino76@gmail.com

1 Ospedale Policlinico San Martino-IRCCS, Genoa, Italy

2 Section of Human Physiology, Department of Experimental Medicine, University of Genoa, Genoa, Italy

3 IRCCS Istituto Delle Scienze Neurologiche di Bologna, Bologna, Italy

4 Department of Biomedical and Neuromotor Sciences, University of Bologna, Bologna, Italy which there are well-founded reasons to consider possible its inappropriate use, which does not bring significant benefits to patients, but rather a greater incidence of side effects or inadequate care. In the selection process, the board of the LIMPEDISMOV Academy took into careful consideration the level of evidence of the practices that was recently revised in the "Linea Guida Diagnosi e Terapia della Malattia di Parkinson" published in 2013 and updated in 2015, drafted by LIMPE in collaboration with the Istituto Superiore di Sanità.

1. Do not use the brain single photon emission tomography with tracers for dopaminergic transporters (DAT-SPECT) for the prognosis and to ascertain the progression of Parkinson's disease.

The DaTscan, a dopamine transporter (DAT) single photon emission computerized tomography (SPECT) imaging technique, proved to be valid in the differential diagnosis between Parkinson's disease and selected neurological conditions (essential tremor, dystonic tremor, and psychogenic parkinsonism). However, several studies have shown that there is insufficient evidence to support the use of DaTscan as a prognostic factor or as a measure of disease progression in Parkinson's disease [1].

2. Do not use antipsychotic medication except clozapine and quetiapine to treat psychosis in Parkinson's disease.

Several studies have shown that clozapine and quetiapine, when used to treat psychosis in Parkinson's disease do not worsen motor symptoms, unlike other antipsychotics. In patients with psychosis and Parkinson's disease, treatment with low-dose clozapine should be considered as the first choice in the treatment of psychosis. However, a mandatory requirement for clozapine use is regular monitoring of white blood 
cell count and absolute neutrophil count. If regular weekly blood tests are not possible, low-dose quetiapine should be considered as an alternative antipsychotic therapy for the treatment of psychosis in Parkinson's disease [2].

3. Do not delay prescribing Levodopa therapy to treat patients early in the course of Parkinson's disease.

Prescription of levodopa as a pharmacological treatment for Parkinson's disease is often delayed in favor of levodopa-sparing therapies (such as dopamine agonists) due to concerns regarding the risk of drug-induced motor complications or possible toxic effect of levodopa. However, the increase in the risk of motor complications associated to levodopa therapy compared to dopamine agonist therapy is still debated, while several studies showed that the use of dopamine agonists increases the incidence of other important side effects (such as impulse control disorders) and induces scarcer control of motor symptoms compared to levodopa. Furthermore, clinical studies have not brought conclusive evidence on the risk of neurotoxicity by early treatment with levodopa. Patients in the early stage of Parkinson's disease may be considered for treatment with levodopa if required by the clinical condition. Noteworthy, the combined treatment of levodopa and entacapone in the early stage of the disease is not indicated, in order to reduce the risk of motor complications [3].

4. Do not use myocardial scintigraphy with metaiodobenzyl guanidine (MIBG) to diagnose Parkinson's disease.

[123I]-MIBG myocardial scintigraphy was originally developed to assess postganglionic presynaptic cardiac sympathetic nerve endings in heart disease. Subsequently, cardiac MIBG uptake was demonstrated to be reduced in patients with Lewy body diseases such as Parkinson's disease. However, several studies have shown that the sensitivity of MIBG myocardial scintigraphy is comparable to that of clinical diagnosis. Therefore, cardiac [123I]-MIBG myocardial scintigraphy may assist in the differential diagnosis of Parkinson's disease versus other parkinsonisms, but it must not be used not to replace clinical diagnosis, especially in the early stages of the disease or in the case of diagnostic uncertainty. Furthermore, particular attention should be paid to the pharmacological treatments of patients, in particular tricyclic antidepressants, which can interfere with the result of the [123I]-MIBG myocardial scintigraphy [4].

5. Do not use anticholinergic drugs to treat the motor symptoms of drug-induced parkinsonisms.

Although anticholinergic drugs have been widely used in the control of parkinsonian symptoms in Parkinson's disease and in parkinsonisms (including drug-induced parkinsonism), the current evidence shows that such drugs have a limited benefit on tremor and are associated with cognitive and neuropsychiatric side effects.

Thus, the use of anticholinergics should be limited in patients with comorbidities as cognitive impairment or clinically significant psychiatric illness. Furthermore, the use of anticholinergic drugs is not recommended for the treatment of motor symptoms in drug-induced parkinsonisms [5].

\section{Conclusions}

The mission of Choosing Wisely is to promote conversations between clinicians and patients by helping patients to choose care that is supported by evidence, not duplicative of other tests or procedures already received, free from harm and truly necessary. The LIMPE-DISMOV Academy as national society with the mission of fighting against Parkinson's disease and of promoting strong interactions between patients and clinicians decided to join with enthusiasm the Choosing Wisely campaign in the hope that the recognition of evidence-based recommendations regarding low-value services in our specialty reduce unnecessary care, avoid harm, and decrease waste.

Future studies will verify if targeting these low-value services and implementing Choosing Wisely recommendations related to tests, treatments, and procedures at risk of inappropriateness in the cure of Parkinson's disease will achieve the expected positive results.

Acknowledgements Collaborators (Board of the LIMPE-DISMOV Academy)

A. Albanese, Department of Neurology, Istituto Clinico Humanitas, Rozzano, Milan, Italy.

A. Antonini, Department of Neurosciences, University of Padua, Padua, Italy.

A. Berardelli, G. Fabbrini, Department of Human Neurosciences, Sapienza, University of Rome and IRCCS Neuromed, Pozzilli, Italy.

C. Colosimo, Department of Neurology, Santa Maria University Hospital, Terni, Italy.

G. Cossu, Department of Neurology, AOB "G. Brotzu” General Hospital, Cagliari, Italy.

G. Defazio, Department of Medical Sciences and Public Health, University of Cagliari, Cagliari, Italy.

L. Lopiano, Department of Neuroscience "Rita Levi Montalcini," University of Turin, Turin, Italy.

F. Mancini, Department of Neurology-Stroke Unit and Laboratory of Neuroscience, IRCCS Istituto Auxologico Italiano, Milan, Italy.

R. Marconi, Unità Operativa Complessa di Neurologia, Ospedale Misericordia, Grosseto, Italy.

F. Morgante, Department of Experimental and Clinical Medicine, University of Messina, Messina, Italy.

M.T. Pellecchia, Center for Neurodegenerative Diseases (CEMAND), Neuroscience Section, University of Salerno, Salerno, Italy.

M. Zappia, Clinica Neurologica I Policlinico Universitario, Catania, Italy. 


\section{Compliance with ethical standards}

Conflict of interest The authors declare that they have no conflict of interest.

Ethical statement This article does not report data collected by any of the authors from human participants or animals.

Open Access This article is distributed under the terms of the Creative Commons Attribution 4.0 International License (http:// creativecommons.org/licenses/by/4.0/), which permits unrestricted use, distribution, and reproduction in any medium, provided you give appropriate credit to the original author(s) and the source, provide a link to the Creative Commons license, and indicate if changes were made.

Publisher's note Springer Nature remains neutral with regard to jurisdictional claims in published maps and institutional affiliations.

\section{References}

1. Kaasinen V, Vahlberg T (2017) Striatal dopamine in Parkinson disease: a meta-analysis of imaging studies. Ann Neurol 82:873-882. https://doi.org/10.1002/ana.25103
2. Seppi K, Weintraub D, Coelho M, Perez-Lloret S, Fox SH, Katzenschlager R, Hametner EM, Poewe W, Rascol O, Goetz CG, Sampaio C (2011) The movement disorder society evidence-based medicine review update: treatments for the non-motor symptoms of Parkinson's disease. Mov Disord 26(Suppl 3):S42-S80. https://doi. org/10.1002/mds.23884 Review

3. Zhang J, Tan LC (2016) Revisiting the medical management of Parkinson's disease: levodopa versus dopamine agonist. Curr Neuropharmacol 14:356-363

4. Orimo S, Suzuki M, Inaba A, Mizusawa H (2012) 123I-MIBG myocardial scintigraphy for differentiating Parkinson's disease from other neurodegenerative parkinsonism: a systematic review and meta-analysis. Parkinsonism Relat Disord 18:494-500. https://doi.org/10. 1016/j.parkreldis.2012.01.009

5. Ferreira JJ, Katzenschlager R, Bloem BR, Bonuccelli U, Burn D, Deuschl G, Dietrichs E, Fabbrini G, Friedman A, Kanovsky P, Kostic V, Nieuwboer A, Odin P, Poewe W, Rascol O, Sampaio C, Schüpbach M, Tolosa E, Trenkwalder C, Schapira A, Berardelli A, Oertel WH (2013) Summary of the recommendations of the EFNS/ MDS-ES review on therapeutic management of Parkinson's disease. Eur J Neurol 20:5-15. https://doi.org/10.1111/j.1468-1331.2012. 03866.x Review 